\title{
Scheduling of Pumped Storage Hydrothermal System with Evolutionary Programming
}

\author{
S. K. Khandualo, A. K. Barisal, and P. K. Hota
}

\begin{abstract}
This paper presents the ever proved evolutionary programming techniques for solving the generation/ pumping scheduling problem of hydro thermal system with pumped storage plants. Pumped storage hydro plant is used to save fuel cost by serving the peak load with hydro energy and then pumping the water back up into the reservoir at light load periods. Therefore, a pumped storage unit can be operated any one mode out of three states such as generation, pumping and idle states. It can smooth peak loads and provide reserves and plays a vital role in reducing the total generation cost in a hybrid power system.
\end{abstract}

Index Terms-Short term hydro scheduling, pumped storage plant, evolutionary programming, and Gradient search.

\section{NOMENCLATURE}

$P_{s i(t)}$ : power generation of thermal unit $i$ in hour $t$

$P h j(t)$ power generating or pumping of $\mathrm{P} / \mathrm{S}$ plant $j$ in hour $t$ positive: generating; negative: pumping

$F_{i(t) P s i(t)}$ : production cost for $P_{s i(t)}$

$T$ : Number of scheduling hours

$N_{h}:$ Number of $\mathrm{P} / \mathrm{S}$ plants

$N_{s}:$ Number of thermal units

$P d(t)$ : System load demand in hour $t$

Ploss $(t)$ : System transmission network losses in hour $t$

$V_{j(t)}$ : Water volume of the upper reservoir of plant $j$ at the ending of hour $t$

$V_{j l(t)}$ :Water volume of the lower reservoir of plant $j$ at the ending of hour $t$

$I_{j(t)}$ : Natural inflow into reservoir $j$ in hour $t$

$Q_{j(t)}$ : Water discharge of $\mathrm{P} / \mathrm{S}$ plant $j$ in hour $t$

$Q_{j t P}$ : Water pumping of $\mathrm{P} / \mathrm{S}$ plant $j$ in hour $t$

$S_{j t}$ : water spillage of $\mathrm{P} / \mathrm{S}$ plant $j$ in hour $t$

\section{INTRODUCTION}

The efficient and optimum economic operation and planning of electric power generation are always vital to the electric power industry. The Short term hydro thermal scheduling problem with a pumped hydro plant is concerned with optimization over a time span of a day or a week. The

Manuscript received October 19, 2012; revised December 18, 2012.

S. K. Khandualo is with the Electrical Engineering Department, Veer Surendra Sai University of Technology, Odisha, India (e-mail: sujatakhandualo@gmail.com).

A. K. Barisal is with the Electrical Engineering Department, VSSUT, Burla, Odisha, India (e-mail: a_barisal@rediffmail.com).

P. K. Hota is with the College of Engineering \& Technology, Bhubaneswar, Odisha, India (e-mail: p_hota@rediffmail.com). pumped hydro plant can be used as a spinning reserve unit to safeguard the system against forced generation outages if the reserve availability is very marginal. The solution to this problem, if the time span is a day gives a plan for the optimal quantity of water to be discharged from or pumped to the pumped hydro plant and the corresponding thermal generation such that the total fuel cost of the thermal plants over the day is minimized subject to the operating constraints of the hydro, pumped hydro and thermal plants.

The Pumped storage hydro plants are designed to save fuel cost i.e during peak load periods; it discharges water for generation at hours of high demand and high costs, displacing high cost fossil generation. During light load periods i.e period of low demand and low cost water is pumped from the lower to the upper reservoir utilizing the economical energy generated by base load units. A pumped storage unit can therefore smooth peak loads, provide reserve, and play an important role in reducing total generation cost.

The optimal scheduling of hydrothermal power system with pumped storage units is usually more complex than that for an all thermal system. It is basically a nonlinear programming problem involving non-linear objective function and a mixture of linear and non-linear constraints. Conventional methods based on Lagrangian multiplier and gradient search techniques require models of hydro as well as thermal plants to be represented as piecewise linear or polynomial approximations of monotonically increasing nature. But such an approximation may lead to suboptimal solution resulting in huge loss of revenue over the time. Methods based on Lagrangian multiplier and gradient search techniques [1] for finding the most economical hydrothermal with pumped storage generation schedule under practical constraints have been well documented. Kirchmayer [2] utilized calculus of variation for short range scheduling problem and proposed the well known coordination equations. In this respect stochastic search algorithms like particle swarm optimization (PSO) [3], simulated annealing (SA) [4], genetic algorithm (GA) [5], evolutionary strategy (ES) [6] and evolutionary programming (EP) [7] may prove to be very efficient in solving highly nonlinear HS problems since they do not place any restriction on the shape of the cost curves and other non-linearities in model representation.

Although these heuristic methods do not always guarantee the globally optimal solution, they will provide a reasonable solution (suboptimal near globally optimal) in a short CPU time. These evolutionary algorithms (EAs) are search algorithms based on the simulated evolutionary process of natural selection and genetics. EAs are more flexible and robust than conventional methods. EP differs from GA in two aspects. Firstly, EP uses the control parameters, but not their 
codings as in GAs. Secondly the generation and selection procedure in EP are mutation and competition but in GAs the procedures are reproduction, mutation and crossover. Hence considerable computation time may thus be saved in EP based on normally distributed mutations.

In recent years, a lot of developments have taken place in EP in terms of efficiency and quality. Here, mutation is the only operator used to generate new offspring. This mutation is implemented by adding to the parent a random number from a certain distribution, e.g. Gaussian distribution in the case of classical EP (CEP). An important parameter of the Gaussian mutation is its standard deviation which is also called strategy parameter. In self-adaptation scheme of EP, this parameter instead of being pre-fixed is evolved along with the objective variables.

\section{Problem Statement}

As hydrogenating units do not incur any fuel cost, the HS problem is aimed to minimize the total thermal cost while making use of the availability of hydro resource as much as possible.

\section{A. Objective Function and Constraints}

The objective function and associated constraints of the problem are formulated as follows. The scheduling of $\mathrm{P} / \mathrm{S}$ units deals with the problem of obtaining the optimal generations both for $\mathrm{P} / \mathrm{S}$ and thermal units. It aims to minimize the production costs of thermal units while satisfying various constraints. With discretization of the total scheduling time into a set of shorter time intervals (say, one hour as one time interval), the scheduling of $\mathrm{P} / \mathrm{S}$ units can be mathematically formulated as a constrained nonlinear optimization problem as follows

$$
\operatorname{Minimize} \sum_{t=1}^{T} \sum_{1}^{N s} F_{i}^{t}\left(P_{s i}^{t}\right)
$$

Subject to the following constraints:

System Power Balance equation:

$$
\sum_{i=1}^{N_{s}} P_{s i}^{t}+\sum_{j=1}^{N_{h}} P_{h j}^{t}-P_{d}^{t}-P_{L}^{t}=0
$$

The Hydro Generation $P_{h j}$ is assumed to be a function of discharge only

$$
P_{h}=f(q)
$$

Thermal Plant generation limit

$$
P_{s i}(\min ) \leq P_{s i}(t) \leq P_{s i}(\max )
$$

Hydro Plant generation limit

$$
P_{h j}(\min ) \leq P_{h j}(t) \leq P_{h j}(\max )
$$

Water Dynamic. Balance

$$
\begin{gathered}
V_{j}^{t}=V_{j}^{t-1}+I_{j}^{t}-Q_{j}^{t}+Q_{j . p}^{t}-S_{j}^{t} \\
V_{j . l}^{t}=V_{j . l}^{t-1}+Q_{j}^{t}-Q_{j . p}^{t}+S_{j}^{t}
\end{gathered}
$$

Water Discharge limits

$$
Q_{j}(\min ) \leq Q_{j}(t) \leq Q_{j}(\max )
$$

Water pumping limits

$$
Q_{j p}(\min ) \leq Q_{j p}(t) \leq Q_{j p}(\max )
$$

Reservoir limits

$$
\begin{gathered}
V_{j}(\min ) \leq V_{j}(t) \leq V_{j}(\max ) \\
V_{j . l}(\min ) \leq V_{j l}(t) \leq V_{j . l}(\max )
\end{gathered}
$$

\section{Evolutionary Programming BASED}

\section{Hydrothermal SCHEdULING FOR PUMPED StORAGE Plant}

Taking the population size to be $I_{p}$, each initial parent trial vector $Q_{i}$, for $\mathrm{i}=1,2, \ldots, I_{p}$ is selected at random from feasible range of each element. To satisfy the constraints on the initial and the final reservoir storage states, a dependent hydro discharge rate $q_{d}$ is randomly selected. The non-dependent hydro discharges $q_{j}$ for $\mathrm{j}=1,2,3, \ldots, \mathrm{J}, \mathrm{j} \neq \mathrm{d}$ are together taken as ( $\mathrm{J}-1)$-dimensional trial vector. Let $Q i=\left[q_{1}, q_{2}, \ldots, q_{(d-1)}, q_{(d+1)}, \ldots q_{j}\right]$ be the trial vector designating the ith individual of a population to be evolved. The selection of each parent trial vector at random is done by setting the jth components of each parent as:

$q_{j}=\operatorname{rand}\left[q_{\min }, q_{\max }\right]$ for $j=1,2, \ldots,(d-1),(d+1), \ldots j$ where rand $\left.\mid q_{\min }, q_{\max }\right\rfloor$ denotes a uniform random variable ranging over $\left[q_{\min }, q_{\max }\right]$ The hydro discharge at dependent interval, $q_{d}$ is calculated from equation (3) with zero spillage (for simplicity) by:

$$
q_{d}=V^{0}-V^{T}-\sum_{\substack{j=1 \\ j \neq d}}^{j} q_{j}+\sum_{j=1}^{j} I_{j}
$$

Knowing hydro discharges, hydrogenations can be calculated from equation (3) by simple algebraic method as the discharge in the present case is a function of hydrogenation. From the calculated hydrogenations $P_{h j}$ and the given load demand $P_{d j}$, for $\mathrm{j}=1,2$, . ., J, power generation of thermal unit in the jth interval $P_{s j}$ can be calculated as:

$$
P_{s j}=P_{d j}+P_{L j}-P_{h j}
$$

Now the thermal production cost and the fitness function which is the sum of production cost and penalty for constraint violation can be calculated for each individual of the parent population as

$$
F I T_{i}=F+\sum_{z=1}^{N c} P F_{z}
$$

And ${ }_{P F_{z}}=\lambda_{z} \times\left[V I O L_{z}\right]^{2}$
$F I T_{i}$ : Fitness value of the ith individual 
$I_{p}$ :No of individuals in the parent population

$I_{p}^{\prime}$ :No of individuals in the child population

j No of scheduling intervals

$P F_{z}$ Penalty associated with the constraint $\mathrm{z}$

$V I O L_{z}$ : amount of violation of constraint $\mathrm{z}$

\section{A. Creation of Offspring}

An offspring is created by mutation as follow:

By Gaussian mutation -An offspring vector $Q i^{\prime}$ is created from each parent $Q_{i}$ by adding to each component of parent, $q_{j}$, a Gaussian random variable with a zero mean and a standard deviation proportional to the scaled cost value of the parent trial solution, i.e.

$$
Q_{i}^{\prime} \text { for } i=1,2 \ldots I_{p} \quad q_{j}^{\prime}=q_{j}+\sigma N(0.1) \text { for } j=1,2, \ldots j
$$

where $N(0,1)$ represents a Normal Gaussian random variable with mean 0 and standard deviation $\sigma=1$ The $\rho$ indicates the range of the off springs created around the parent trial vector and their expressions in case of scaled cost are given by:

$$
\sigma=\beta \times F I T_{i} / F I T_{\min }\left(q_{\max }-q_{\min }\right)
$$

where $F I T$ min is the minimum value of fitness among the $I_{p}$ trial solutions and $\beta$ is scaling factor.

TABLE I: LOAD PATTERN OF THE TEST SySTEM

\begin{tabular}{|c|c|c|}
\hline Interval number & Interval & Demand (MW) \\
\hline 1 & 00:00_04:00 h & 1600 \\
\hline 2 & 04:00_08:00 h & 1800 \\
\hline 3 & 08:00_12:00 h & 1600 \\
\hline 4 & 12:00_16:00 h & 500 \\
\hline 5 & 16:00_20:00 h & 500 \\
\hline 6 & 20:00_24:00 h & 500 \\
\hline
\end{tabular}

TABLE-II : TUNED PARAMETER SETTINGS OF THE EPS

\begin{tabular}{|c|c|}
\hline Control parameters & Value \\
\hline Scaling factor $(\beta)$ & 0.01 \\
\hline Population size & 10 \\
\hline Maximum Iteration & 250 \\
\hline
\end{tabular}

\section{B. Competition and Selection}

After generation of offspring population, competition and selection procedure is implemented to determine which solutions are to be retained into the next generation and which are to be removed from the competiting pool of trials. The parent trial vectors $Q_{i}$,for $i=1,2, \ldots I_{p}$ and their corresponding off springs $Q_{i}{ }^{\prime}$ contend with each other for survival within the competiting pool. In this process a competitor $Q_{r}$ is selected at random from among the $2 I_{p}$ trial solutions, where ' $\mathrm{r}$ ' is an integer as given by: $r \approx\left[2 I_{p} \times u_{1}+1\right]$ here $\mathrm{r}$ is taken to be the greatest integer less than or equal to the value of the expression in the right hand side.

A weight value $\mathrm{w}^{\mathrm{r}}$ is assigned to each individual as follows

$$
w_{Q_{i}}=\sum_{m=1}^{I_{p}} w_{m}
$$

And a

$$
\mathcal{W}_{m}=1, \text { if } u_{2} \leq F I T \lambda / F I T_{I}+F I T \lambda
$$

$w_{m}=0$, otherwise

where $u 1$ and $u 2$ are uniform random numbers ranging over [0.1].

After the competition is over, the $2 I_{p}$ trial solutions are sorted in descending order of the score obtained in equation (15). The first $I_{p}$ trial solutions from the sorted pool are selected as the new parent vectors for the next generation.

\section{IMPLEMENTATION OF EP IN HYDROTHERMAL SCHEDULING}

Step 1: The problem variables to be determined are represented as a J-dimensional trial vector, where each vector is an individual of the population to be evolved.

Step 2: An initial population of parent vectors, $Q_{i}$, for i_ $1,2, \ldots, I_{p}$, is selected at random from a feasible range in each dimension. The distribution of these initial parent vectors is uniform.

Step 3:An offspring $\left(Q_{i}{ }^{\prime}\right)$ is generated from each parent by Gaussian mutation (CEP), as depicted in Section 3 with adaptation of strategy parameter, $\mathrm{s}$ based on scaled cost.

Step 4: Fitness function, $F I T_{i}$ is evaluated for each individual of both parent and child populations.

Step 5: A competitor is chosen randomly from the combined population of $2 I_{p}$ trial solutions ( $I_{p}$ parent and $I_{p}^{\prime} \quad$ offspring) and stochastic competition is performed based on the value of fitness function where each individual in the competing pool compete against other members for survival.

Step 6: After the competition is over, the $2 I_{p}$ trial solutions in the competing pool are sorted according to their scores from the highest to the lowest. There after the first $I_{p}$ trial solutions are selected as the new parent vectors for the next generation.

Step 7: If current generation is greater than or equal to the maximum generation, stop and print the result. Otherwise repeat the steps 3 to 6 .

\section{TEST SySTEM}

The performance of the proposed EP algorithms is verified on a test system which has been adopted from [1]. It comprises a pumped storage hydro and an equivalent thermal plant. The schedule horizon is 1 day and there are six $4 \mathrm{~h}$ intervals. The load pattern showing the load demands in the intervals are given in Table 1. 
The fuel cost function of the equivalent thermal unit is:

$$
F\left(P_{s}\right)=575+9.2 P_{s}+0.00184 P_{s}^{2}
$$

$\left(200 m w \leq P_{s} \leq 2500 m w\right)$

The pumped storage plant has the following characteristics Generating

$$
\begin{aligned}
& q \text { Positive when generating, } P_{H} \text { is positive } \\
& 0 \leq P_{H} \leq+300 M W \\
& q\left(P_{H}\right)=200+2 P_{H} \quad \text { acreft } / \mathrm{hr}
\end{aligned}
$$

Pumping: q negative when pumping, $P_{p}$ is negative and $-300 M W \prec P_{p} \leq 0 M W \quad, \quad q\left(P_{p}\right)=-600 \quad$ acreft $/ \mathrm{h} \quad$ with $P_{p}=-300 M W$.

Operating restrictions: The pumped hydro plant will be allowed to operate only at $-300 \mathrm{mw}$ when pumping. Cycle efficiency is 0.6667 [The efficiency has already been built into the $q\left(P_{H}\right)$ equations].

The reservoir starts at 8000 acre $-f t$ and must be at 8000 acre $-f t$ at the end of the sixth period. The water inflow rate is assumed to be neglected and the spillage is not counted. Further, the electrical loss from the hydro plant to the load is taken to be negligibly small.

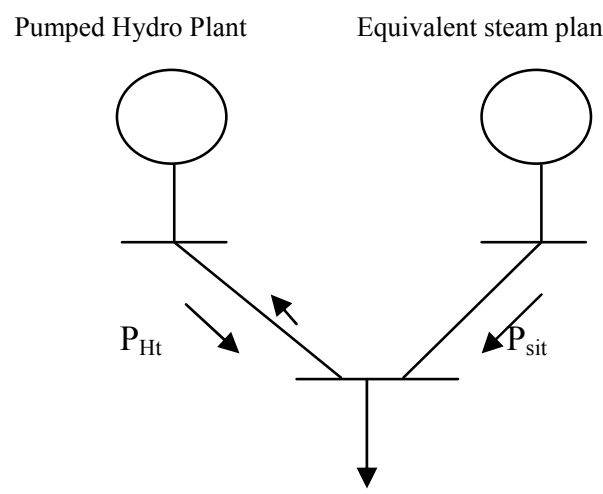

Fig. 1. Schematic diagram of test system

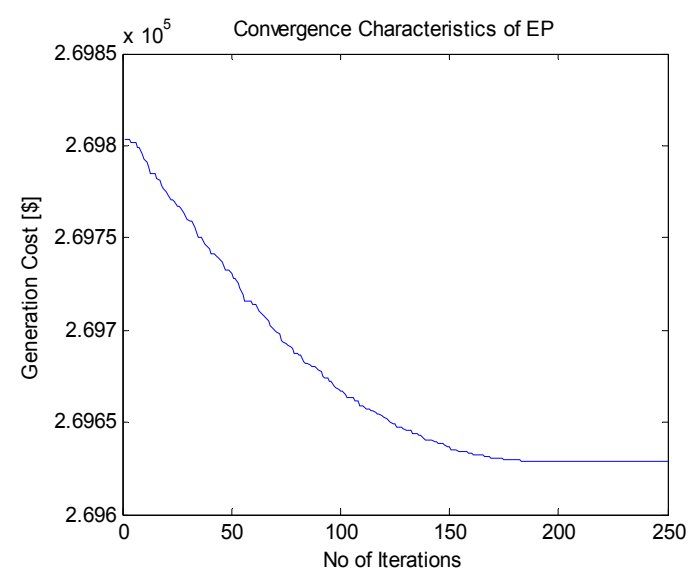

Fig. 2. Convergence characteristics of EP algorithm.

\section{Simulation Results}

All the programs were implemented in MATLAB command line on a PC (Pentium-III, 4GB). The control parameters in the EP programs used for solving the test case were tuned and their tuned values are listed in Table II. The HS and the system costs obtained from proposed EP and the gradient search [1] are summarized in Table III.

The optimal system costs obtained from EP are given in the table. It can be seen from the table that the optimal cost

\begin{tabular}{|c|c|c|c|c|c|c|}
\hline \multicolumn{7}{|c|}{ PLANT } \\
\hline 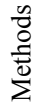 & $\begin{array}{l}\text { Int. } \\
\text { No }\end{array}$ & $\begin{array}{l}\text { Thermal } \\
\text { Power }\end{array}$ & $\begin{array}{l}\text { Hydro } \\
\text { Power }\end{array}$ & $\begin{array}{l}\text { Volume } \\
\text { Acre } \mathrm{ft}\end{array}$ & $\begin{array}{c}\mathrm{Q} \\
\text { Acreft } / \mathrm{hr}\end{array}$ & Cost \\
\hline \multirow{6}{*}{ EP } & 1 & 1466.4211 & 133.5789 & 6131.37 & 467.16 & \multirow{6}{*}{$\begin{array}{r}269628 . \\
8\end{array}$} \\
\hline & 2 & 1466.9846 & 333.0154 & 2667.25 & 866.03 & \\
\hline & 3 & 1466.5943 & 133.4057 & 800 & 466.81 & \\
\hline & 4 & 800 & -150 & 3200 & -600 & \\
\hline & 5 & 800 & -300 & 5600 & -600 & \\
\hline & 6 & 800 & -300 & 8000 & -600 & \\
\hline \multirow{6}{*}{ GS } & 1 & 1450 & 150 & 6000 & 500 & \multirow{6}{*}{$\begin{array}{r}269642 . \\
4\end{array}$} \\
\hline & 2 & 1500 & 300 & 2800 & 800 & \\
\hline & 3 & 1450 & 150 & 800 & 500 & \\
\hline & 4 & 800 & -300 & 3200 & -600 & \\
\hline & 5 & 800 & -300 & 5600 & -600 & \\
\hline & 6 & 800 & -300 & 8000 & -600 & \\
\hline
\end{tabular}
obtained by the proposed EP is the lower than the Gradient Search technique.

TABLE III: THE SCHEDULING OF PUMPED STORAGE HYdROTHERMAL

\section{CONCLUSION}

Evolutionary programs with Gaussian mutation was developed and demonstrated to solve the pumped storage Hydro scheduling problem. Results show that EP-based algorithms are more capable of finding highly near-global solutions than Gradient Search techniques. The optimal cost obtained by the Evolutionary Programming is quite cheaper than the Gradient Search technique for the system adopted. In future, attempts can be made to apply the Evolutionary programming technique to Pumped storage unit in conjunction with wind energy by incorporating emission, spinning reserve and reliability constraints.

\section{REFERENCES}

[1] A. J. Wood and B. F. Wollenberg, Power Generation, Operation and Control, $2^{\text {nd }}$ ed, New York: Wiley, 1984, ch. 7, pp. 230-239.

[2] S. Sivanagaraju and G. Srinivasan, Power System Operation and Control, Pearson Education India Ltd. 2010, pp. 218-222.

[3] P. H. Chen, C. C. Kuo, F. H. Chen, and C. C. Chen "Refined Binary Particle Swarm Optimization and Application in Power System," WSEAS Transactions on Systems, vol. 8, no. 2, pp. 169-178, February 2009.

[4] K. P. Wong and Y. W. Wong, "Short-term hydrothermal scheduling, part-I: simulated annealing approach," IEE Proc. Part-C, vol. 141, no. 5, pp. 497-501, 1994.

[5] Y. G. Wu, C. Y. Ho, and D. Y. Wang, "A diploid genetic approach to short-term scheduling of hydrothermal system," IEEE Trans. Power Syst, vol. 15, no. 4, pp. 1268-1274, 2000.

[6] T. Ba"ck and H. P. Schwefel, "An overview of evolutionary algorithms for parameter optimization," Evol. Comput. vol. 1, no. 1, 1993, pp. $1-23$.

[7] D. P. Kothari and J. S. Dhillon, Power System Optimization, India: PHI Learning Pvt Ltd, 2011, pp. 547-552.

[8] N. Sinha, R. Chakrabarti, and P. K. Chattopadhyay, "Fast evolutionary programming techniques for short-term hydrothermal scheduling," Elect. Power syst. Res., vol. 66, no. 2, pp. 97-103, August 2003. 


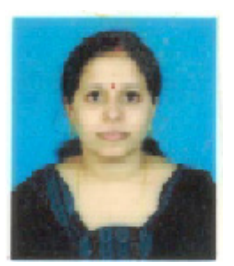

Sujata Kumari Khandualo was born in 1979 and received the B.Tech. degree from the C.E.T, Bhubaneswar, Odisha, in 2003 and pursuing M. Tech. degree in power system engineering in the Electrical Engineering Department, V.S.S University of Technology, Burla, Odisha, India.

She was working as Asst Manager (El) at Chiplima Hydro Electric Project under Odisha Hydro Power Corporation Ltd, Bhubaneswar. Her research interests include Hydrothermal Scheduling and soft computing applications to power system problems.

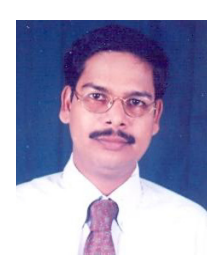

Ajit Kumar Barisal was born in 1975 and received the B.E. degree from the U.C.E, Burla (now VSSUT), Odisha,India in 1998 and the M.E.E. degree in power system from Bengal engineering College (now BESU), Shibpur, Howrah, in 2001 and $\mathrm{Ph}$. D degree from Jadavpur University, Kolkata in 2010, all in electrical engineering. He was with the Electrical Engineering Department, NIST, Berhampur, Orissa, from 2000 to 2004 and with Electrical and Electronics Engineering Department, Silicon Institute of Technology, Bhubaneswar, Orissa, from 2004 to 2005. Since 2006, he has been with the Electrical Engineering Department, V.S.S University of Technology, Burla, Odisha, where he is a Reader. He received the "Odisha Young Scientist award- 2010 ”, IEI Young Engineers award2010 " and "Union Ministry of Power, Department of power prize- 2010" for his outstanding contribution to Engineering and Technology research. His research interests include economic load dispatch, Hydrothermal Scheduling, alternative energy power generation and soft computing applications to different power system problems.

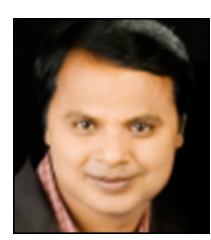

Prakash Kumar Hota was born in 1963 in India and currently the Principal of College of Engineering and Technology (CET), Bhubaneswar and Dean Faculty of Engineering and Faculty of Architecture, Biju Patnaik University of Technology, Odisha, had his graduation in Electrical and Electronics Engineering from the National Institute of Technology (NIT), Tiruchillapali , India in 1985. After a brief stint in private sector joined as lecturer in the Department of Electrical Engineering of University College of Engineering Burla, Orissa in 1988 and became Reader in 1997 and also Professor in Training \& placement of UCE Burla in 2006. He has been selected to be listed in "The Contemporary WHO's WHO" 2002-2003 published by The American Biographical Institute, USA on the basis of merited accomplishment and success in contemporary Society. He received the "Pandit Madan Mohan Malaviya award" by IE, India, "Rajlaxmi Memorial best engineering college teacher award by ISTE, New-Delhi" and "Union Ministry of Power, Department of power prize" for his outstanding contribution to Engineering and Technology research. He was the Dean, Students Welfare in VSS University of Technology from 2009-2011. His research interests include economic emission load dispatch, Hydrothermal Scheduling, Wind power generation, Power Quality and soft computing applications to different power system problems in deregulated environment. 